\title{
Conhecimento sobre cárie dentária entre docentes do ensino médio
}

\author{
Gabriele Amorim Cordeiro*; Manoelito Ferreira Silva-Junior**; Luiz Gustavo Dias Daroz***; Cláudia \\ Batitucci dos Santos Daroz***
}

* Graduanda em Odontologia, Universidade Federal do Espírito Santo

** Professor Colaborador, Departamento de Odontologia, Universidade Estadual de Ponta Grossa

*** Professor Adjunto, Departamento de Prótese Dentária, Universidade Federal do Espírito Santo

Recebido em 12/07/2018. Aprovado em 22/12/2019.

\begin{abstract}
RESUMO
O objetivo do estudo foi avaliar o conhecimento sobre cárie dentária entre docentes do ensino médio. Trata-se de um estudo exploratório transversal com abordagem quantitativa entre docentes do ensino médio de escolas públicas e privadas do município de Vitória/ES. O estudo utilizou um questionário autoaplicado validado, adaptado para população-alvo, com perguntas que versavam sobre o conhecimento da cárie. Os dados descritivos foram apresentados em frequências absolutas e relativas e houve o teste de associação utilizando o teste Exato de Fisher ( $<<0,05)$. Um total de 38 professores participaram do estudo, sendo $25(65,8 \%)$ de escolas públicas. A maioria dos professores não recebeu informações sobre cárie $(60,5 \%)$ durante sua formação acadêmica e seus conhecimentos foram adquiridos nas visitas ao cirurgião-dentista particular (50,0\%). A maior parte soube definir cárie como doença $(71,1 \%)$, no entanto, com incertezas $(39,5 \%)$ se a lesão era a principal consequência do seu desenvolvimento e obrigatoriedade de restauração $(55,3 \%)$ para o seu tratamento. O tema cárie é abordado em sala de aula por $60,5 \%$ dos docentes, principalmente no segundo ano $(60,9 \%)$ e nas escolas particulares $(\mathrm{p}=0,0285)$. Dos professores, a maior parte gostaria que o tema cárie fizesse parte da estrutura curricular do ensino médio $(92,1 \%)$ por acreditaram na relevância do assunto $(97,4 \%)$. A maioria dos docentes soube definir a cárie como uma doença, no entanto, houve relativo desconhecimento de suas consequências e formas de tratamento.

Descritores: Cárie Dentária. Educação em Saúde. Ensino Fundamental e Médio.
\end{abstract}

\section{INTRODUÇÃO}

A percepção que um indivíduo tem sobre uma determinada doença pode alterar-se de acordo com as fontes de informação e experiências pessoais. Informações passadas pelos familiares, obtidas nos diversos meios de comunicação como a televisão, rádio ou internet, aquelas passadas por profissionais da área da saúde da rede pública ou particular, e as aprendidas durante a vida escolar do indivíduo são exemplos de fontes de informação que podem 
contribuir para o entendimento sobre diversos aspectos de saúde e prevenção de doenças. A educação e motivação são capazes de despertar interesse pela manutenção da saúde, desenvolvendo nas pessoas consciência crítica das reais causas de seus problemas ${ }^{1}$.

Como a higiene é essencial para uma boa saúde bucal, é necessário fornecer à população orientações adequadas sobre o comportamento relacionado à saúde bucal e sua relação com a cárie dentária ${ }^{2}$. Assim, é essencial o trabalho conjunto entre profissionais de saúde e da educação, atuando como colaboradores dos programas educativospreventivos $^{3}$.

O ambiente escolar é um espaço propício para que conceitos de saúde bucal sejam ensinados de forma ampla e contínua, pois agrupa crianças e adolescentes em faixas etárias propícias à adoção de medidas educativas e preventivas ${ }^{4}$. Idealmente, esse conhecimento, bem como de outras enfermidades que se constituem transtornos a saúde pública, deveriam acompanhar o indivíduo durante toda sua formação escolar. Todavia, a literatura encontrada é escassa sobre o conhecimento adquirido durante a formação no ensino fundamental e médio sobre a cárie e suas implicações nos hábitos de higiene e autocuidado bucal ${ }^{5-10}$.

Os professores do ensino básico desempenham papel fundamental enquanto multiplicadores de conhecimentos e podem se tornar agentes motivadores e promotores de saúde no ensino e contextualização do autocuidado, inclusive em saúde bucal ${ }^{10}$. Nesse caminho, uma atuação interdisciplinar, com a aproximação entre os cursos de Odontologia e cursos de licenciatura, pode ser uma ferramenta fundamental na transformação do ensino-aprendizagem dos escolares ${ }^{11}$.

Sendo assim, é de suma importância avaliar o conhecimento de docentes a respeito da doença cárie para entender o processo ensino-aprendizagem dos alunos que estão recebendo essas informações. $\mathrm{O}$ objetivo do presente estudo foi avaliar o conhecimento sobre cárie dentária entre docentes de ensino médio.

\section{METODOLOGIA}

Esse estudo do tipo exploratório transversal com abordagem quantitativa foi realizado por meio de um questionário estruturado validado ${ }^{9}$, com docentes do ensino médio da área de ciências biológicas de escolas públicas e particulares do município de Vitória/ES.

O presente estudo faz parte do Projeto Inserção do Tema "cárie dental" no Sistema Educacional Brasileiro e foi aprovado no Comitê de Ética em Pesquisa Centro de Ciências da Saúde da Universidade Federal do Espírito Santo (CEP-CCS-Ufes) sob o parecer número 1.616.366 (CAAE: 56210416.8.0000.5060).

A população alvo do presente estudo consistiu em professores da área de ciências biológicas do ensino médio de 12 escolas públicas $(n=36)$ e de 11 escolas particulares $(n=44)$, totalizando 80 docentes da cidade de Vitória/ES.

Todos os questionários foram entregues à diretoria de cada escola, distribuídos e recolhidos pelos coordenadores de cada ano. Os participantes preencheram sozinhos o seu questionário e os mesmos foram recolhidos nas escolas pela própria pesquisadora.

O instrumento utilizado foi previamente validado por Santos-Daroz et al. ${ }^{9}$ com estudantes de Odontologia, por isso houve uma adaptação referente às características do público-alvo do estudo. $\mathrm{O}$ questionário possibilitava a caracterização da amostra (escola, ano de ensino, sexo, idade, formação do professor, entre outros) e abordava questões referentes ao conhecimento da doença cárie dentária (tais como definição, consequência e métodos de prevenção) e local onde adquiriu o conhecimento (graduação, casa, escola, cirurgião-dentista, dentre outros). 
Os dados foram tabulados no programa Excel 2016® (Microsoft Corporation, Redmond, Washington, USA) e foi realizada análise descritiva com frequência absoluta (n) e relativa (\%). Para análise de associação foi utilizado o teste Exato de Fisher $(\mathrm{p}<0,05)$.

\section{RESULTADOS}

Duas escolas particulares e uma pública se recusaram a participar da pesquisa. Assim, um total de 69 questionários foram entregues. A taxa de devolução foi de $55 \%$, totalizando em amostra com 38 professores (25 de escolas públicas e 13 de escolas particulares).

No presente estudo houve maior participação de professores do sexo feminino $(55,3 \%)$, com idade entre 30 e 39 anos (52,6\%), casados (47,4\%), com renda familiar entre 5 e 6 salários mínimos $(34,2 \%)$. A formação desses professores se deu durante o ensino fundamental $\left(6^{\circ}\right.$ ao $9^{\circ}$ ano) na rede pública $(57,9 \%)$, o ensino médio ( $1^{\circ}$ ao $3^{\circ}$ ano) na rede privada $(57,9 \%)$, retornando para a rede pública $(57,9 \%)$ durante a graduação, com curso superior em Ciências Biológicas (89,5\%), sendo que 81,6\% desses professores cursaram pós-graduação (tabela 1). A maioria dos professores $(36,8 \%)$ leciona para todos os anos do ensino médio (tabela 2).

A respeito dos conhecimentos sobre a cárie, apenas 39,5\% afirmam ter recebido informações sobre o processo saúde-doença durante a graduação, no entanto $50,0 \%, 42,1 \%$ e $28,9 \%$ acreditam que a maior parte do conhecimento foi advindo do cirurgião-dentista particular, de casa e da escola, respectivamente. A totalidade dos docentes afirma que após adquirirem os conhecimentos sobre a doença cárie conseguiram aplicá-los em sua prática diária, proporcionando melhoras em sua saúde bucal (tabela 3). Foi observado que $71,1 \%$ dos participantes caracterizaram a cárie como uma doença, sendo sua consequência uma lesão para $39,5 \%$ e perda dentária para $31,6 \%$. Para $55,3 \%$ dos docentes, toda cárie precisa ser restaurada/obturada (tabela 4).

Em relação aos métodos de prevenção da cárie, todos os entrevistados afirmaram conhecer a escovação caseira regular, seguida do uso de fio dental $(97,4 \%)$ e visita periódica ao dentista (94,7\%), já cuidados com a alimentação e uso de bochechos com flúor foram relatados por $84,2 \%$ e $73,7 \%$ dos respondentes, respectivamente. Quando questionados quais dos métodos preventivos da cárie são utilizados por eles, os resultados obtidos foram: escovação caseira $(97,4 \%)$; uso do fio dental (97,4\%); uso de bochechos com flúor (55,3\%); e cuidados com a alimentação (50,0\%). A maioria dos docentes $(76,3 \%)$ responderam que visitam regularmente o cirurgião-dentista, uma vez ao ano $(47,4 \%)$ (tabela 4).

Em sala de aula, 60,5\% aborda o assunto cárie, em sua maioria no $2^{\circ}$ ano do ensino médio $(60,9 \%)$ (tabela 5), sendo o tema menos abordado por docentes das escolas públicas $(\mathrm{p}=0,0285)$. Quase a totalidade dos docentes $(97,4 \%)$ consideram o tema cárie importante e gostariam $(92,1 \%)$ que fizesse parte da estrutura curricular do ensino médio, pois $100 \%$ acreditam que com a inclusão desse conteúdo em sala de aula a incidência de cárie diminuiria. Contudo, 60,5\% acreditam que o conteúdo cárie e seus métodos de prevenção deveriam ser adotados no ensino fundamental e médio como matéria lecionada (tabela 5).

\section{DISCUSSÃO}

No presente estudo a maioria dos professores acha ser importante e gostaria que o conteúdo cárie e seus métodos de prevenção fizesse parte da estrutura curricular do ensino médio. Percebeu-se que, apesar da maioria dos professores terem conhecimento sobre a doença cárie e seus métodos de prevenção, pouco mais da metade transmite seus conhecimentos aos alunos, provavelmente porquê este não seja um assunto obrigatório na estrutura curricular a ser ofertada pelas escolas ou a ausência dessa temática nos cursos de licenciatura. 
Tabela 1. Caracterização da amostra

\begin{tabular}{|c|c|c|}
\hline Variáveis & $\mathbf{n}$ & $\%$ \\
\hline \multicolumn{3}{|l|}{ Sexo } \\
\hline Masculino & 17 & 44,7 \\
\hline Feminino & 21 & 55,3 \\
\hline \multicolumn{3}{|l|}{ Faixa etária } \\
\hline De 24 a 29 anos & 7 & 18,4 \\
\hline De 30 a 39 anos & 20 & 52,6 \\
\hline De 40 a 49 anos & 5 & 13,2 \\
\hline De 50 a 59 anos & 4 & 10,5 \\
\hline De 60 a 66 anos & 2 & 5,3 \\
\hline \multicolumn{3}{|l|}{ Estado civil } \\
\hline Solteiro & 17 & 44,7 \\
\hline Casado & 18 & 47,4 \\
\hline Divorciado & 3 & 7,9 \\
\hline \multicolumn{3}{|l|}{ Renda familiar } \\
\hline De 1 a 2 salários mínimos & 1 & 2,6 \\
\hline De 3 a 4 salários mínimos & 10 & 26,3 \\
\hline De 5 a 6 salários mínimos & 13 & 34,2 \\
\hline De 7 a 8 salários mínimos & 5 & 13,2 \\
\hline Mais de 8 salários mínimos & 7 & 18,4 \\
\hline Não respondeu & 2 & 5,3 \\
\hline \multicolumn{3}{|c|}{$\begin{array}{l}\text { Cursou o ensino fundamental }\left(\text { de }^{\circ} \text { ao } 9^{\circ} \text { ano) em uma }\right. \\
\text { instituicão }\end{array}$} \\
\hline Pública & 22 & 57,9 \\
\hline Privada & 13 & 34,2 \\
\hline Pública e Privada & 3 & 7,9 \\
\hline \multicolumn{3}{|c|}{ Cursou o ensino médio (de $1^{\circ}$ ao $3^{\circ}$ ano) em uma instituição } \\
\hline Pública & 13 & 34,2 \\
\hline Privada & 22 & 57,9 \\
\hline Pública e Privada & 3 & 7,9 \\
\hline \multicolumn{3}{|c|}{ Cursou o ensino superior em uma instituição } \\
\hline Pública & 22 & 57,9 \\
\hline Privada & 14 & 36,8 \\
\hline Pública e Privada & 2 & 5,3 \\
\hline \multicolumn{3}{|l|}{ Formação acadêmica } \\
\hline Ciências Biológicas & 34 & 89,5 \\
\hline Ciências Biológicas e Medicina & 1 & 2,6 \\
\hline Ciências Biológicas e Pedagogia & 1 & 2,6 \\
\hline Gestão Ambiental & 1 & 2,6 \\
\hline Medicina Veterinária & 1 & 2,6 \\
\hline \multicolumn{3}{|l|}{ Possui Pós-graduação } \\
\hline Não & 7 & 18,4 \\
\hline Sim & 31 & 81,6 \\
\hline Total & 38 & 100,0 \\
\hline
\end{tabular}


Tabela 2. Distribuição absoluta e relativa das turmas do ensino médio que os professores lecionam

\begin{tabular}{lcc}
\hline Variável & n & \% \\
\hline Turmas de ensino médio que leciona & & \\
$1^{\mathbf{o}}$ ano & 3 & 7,9 \\
$2^{\mathbf{o}}$ ano & 3 & 7,9 \\
$3^{\mathbf{o}}$ ano & 8 & 21,0 \\
Todas as turmas & 14 & 36,8 \\
$1^{\mathbf{o}}$ e $2^{\circ}$ anos & 3 & 7,9 \\
$1^{\mathbf{o}}$ e $3^{\circ}$ anos & 4 & 10,5 \\
$2^{\mathbf{o}}$ e $3^{\circ}$ anos & 3 & 7,9 \\
\hline Total & $\mathbf{3 8}$ & $\mathbf{1 0 0 , 0}$ \\
\hline
\end{tabular}

Tabela 3. Distribuição absoluta e relativa sobre a temporalidade do conhecimento sobre a cárie

\begin{tabular}{|c|c|c|}
\hline Variáveis & $\mathbf{n}$ & $\%$ \\
\hline \multicolumn{3}{|l|}{$\begin{array}{l}\text { No ensino superior, você recebeu alguma informação sobre o } \\
\text { processo saúde-doença, a respeito da cárie: }\end{array}$} \\
\hline Não & 23 & 60,5 \\
\hline Sim & 15 & 39,5 \\
\hline \multicolumn{3}{|l|}{$\begin{array}{l}\text { Você acredita que a maior parte de seus conhecimentos sobre a cárie } \\
\text { veio de*: }\end{array}$} \\
\hline Casa & 16 & 42,1 \\
\hline Escola & 11 & 28,9 \\
\hline Dentista particular & 19 & 50,0 \\
\hline Serviço Público & 2 & 5,3 \\
\hline Meios de comunicação & 3 & 7,9 \\
\hline Da Graduação & 2 & 5,3 \\
\hline Outros (Pesquisas e Pesquisa pessoal) & 2 & 5,3 \\
\hline \multicolumn{3}{|l|}{$\begin{array}{l}\text { Quando você adquiriu esses conhecimentos, conseguiu aplicá-los a } \\
\text { suas práticas diárias? }\end{array}$} \\
\hline Não & - & - \\
\hline Sim & 38 & 100,0 \\
\hline \multicolumn{3}{|l|}{$\begin{array}{l}\text { Quando você adquiriu esses conhecimentos, acredita que essas } \\
\text { informações proporcionaram melhora de sua saúde bucal? }\end{array}$} \\
\hline Não & - & - \\
\hline Sim & 38 & 100,0 \\
\hline Total & 38 & $\mathbf{1 0 0 , 0}$ \\
\hline
\end{tabular}


Tabela 4. Distribuição absoluta e relativa sobre o conhecimento dos professores sobre a cárie

\begin{tabular}{lcc}
\hline \multicolumn{1}{c}{ Variáveis } & n & \% \\
\hline O que é a cárie dentária? & & \\
Um bichinho que come o dente & 27 & - \\
Uma doença & 9 & 23,1 \\
Consequência a uma doença & 2 & 5,3 \\
É a falta de escovar o dente & & \\
\hline Toda cárie precisa ser restaurada/obturada? & 21 & 55,3 \\
Sim & 17 & 44,7 \\
Não & \multicolumn{3}{c}{} \\
\hline Qual é a consequência do desenvolvimento da cárie? & 2 & 5,3 \\
Um buraco no dente & 15 & 39,5 \\
Uma lesão & 9 & 23,7 \\
Desgaste dentário & 12 & 31,6 \\
Perda dentária
\end{tabular}

Marque qual(ais) do(s) método(s) de prevenção contra cárie, abaixo, você conhece*:

$\begin{array}{lll}\text { Escovação caseira regular } & 38 & 100,0\end{array}$

$\begin{array}{lll}\text { Uso de fio dental } & 37 & 97,4\end{array}$

$\begin{array}{lll}\text { Visita periódica ao dentista } & 36 & 94,7\end{array}$

$\begin{array}{lll}\text { Cuidados com a alimentação } & 32 & 84,2\end{array}$

$\begin{array}{lll}\text { Uso de bochechos com flúor } & 28 & 73,7\end{array}$

$\begin{array}{lll}\text { Outros (Bochecho com água oxigenada 10V) } & 1 & 2,6\end{array}$

Marque quais dos métodos de prevenção abaixo você utiliza*:

$\begin{array}{lll}\text { Escovação caseira regular } & 37 & 97,4\end{array}$

$\begin{array}{lll}\text { Uso de fio dental } & 37 & 97,4\end{array}$

$\begin{array}{lll}\text { Uso de bochechos com flúor } & 21 & 55,3\end{array}$

$\begin{array}{lll}\text { Cuidados com a alimentação } & 19 & 50,0\end{array}$

$\begin{array}{lll}\text { Outros (Bochecho com água oxigenada 10V) } & 1 & 2,6\end{array}$

Você visita regularmente o dentista?

$\begin{array}{lll}\text { Não } & 9 & 23,7\end{array}$

$\begin{array}{lll}\operatorname{Sim} & 29 & 76,3\end{array}$

\begin{tabular}{lccc}
\hline $\begin{array}{l}\text { Em qual das situações abaixo você procura um cirurgião- } \\
\text { dentista: }\end{array}$ & & \\
Só em casos de dor & 6 & 15,8 \\
Uma vez ao ano & 18 & 47,4 \\
Semestralmente & 8 & 21,1 \\
Para tratar cárie & - & - \\
Para limpeza e prevenção & 3 & 7,9 \\
Emergência & 3 & 7,9 \\
\hline Total & $\mathbf{3 8}$ & $\mathbf{1 0 0 , 0}$ \\
\hline * Mais de uma opção poderia ser assinalada. & &
\end{tabular}


Tabela 5. Distribuição absoluta e relativa sobre o ensino em sala de aula sobre a temática cárie

\begin{tabular}{|c|c|c|}
\hline Variáveis & $\bar{n}$ & $\%$ \\
\hline \multicolumn{3}{|l|}{ O conteúdo da cárie é falado durante sua aula: } \\
\hline Não & 15 & 39,5 \\
\hline Sim & 23 & 60,5 \\
\hline \multicolumn{3}{|l|}{ Se respondeu "SIM", em qual ano? } \\
\hline $1^{\circ}$ ano & 8 & 34,8 \\
\hline $2^{\circ}$ ano & 14 & 60,9 \\
\hline $3^{\circ}$ ano & 10 & 43,5 \\
\hline \multicolumn{3}{|c|}{$\begin{array}{l}\text { Você acredita que com a inclusão desse conteúdo em sala de aula a } \\
\text { incidência da cárie: }\end{array}$} \\
\hline Diminuiria & 38 & 100,0 \\
\hline Aumentaria & - & - \\
\hline Se manteria & - & - \\
\hline \multicolumn{3}{|c|}{$\begin{array}{l}\text { Você acha importante que o assunto cárie faça parte da estrutura } \\
\text { curricular do ensino médio: }\end{array}$} \\
\hline Não & 1 & 2,6 \\
\hline Sim & 37 & 97,4 \\
\hline \multicolumn{3}{|c|}{$\begin{array}{l}\text { Você gostaria que o assunto cárie fizesse parte da estrutura } \\
\text { curricular do ensino médio: }\end{array}$} \\
\hline Não & 2 & 5,3 \\
\hline Sim & 35 & 92,1 \\
\hline Não respondeu & 1 & 2,6 \\
\hline \multicolumn{3}{|c|}{$\begin{array}{l}\text { Em que série você acredita que a cárie e seus métodos de prevenção } \\
\text { deveriam ser adotados no ambiente escolar como matéria lecionada? }\end{array}$} \\
\hline De $1^{\mathrm{a}}$ a $4^{\mathrm{a}}$ série & 7 & 18,4 \\
\hline De $5^{\mathrm{a}}$ a $8^{\mathrm{a}}$ série & 5 & 13,2 \\
\hline No ensino médio $\left(1^{\circ}, 2^{\circ}\right.$ e $3^{\circ}$ ano $)$ & - & - \\
\hline No ensino fundamental e médio & 23 & 60,5 \\
\hline Não acho importante a temática em sala de aula & 1 & 2,6 \\
\hline De $1^{\mathrm{a}}$ a $4^{\mathrm{a}}$ série $/ 5^{\mathrm{a}}$ a $8^{\mathrm{a}}$ série & 2 & 5,3 \\
\hline Total & 38 & 100,0 \\
\hline
\end{tabular}

Apesar da formação desses professores ser especialmente em Ciências Biológicas (89,5\%), a maioria $(60,5 \%)$ relatou não ter recebido nenhuma informação sobre o processo saúde-doença relacionado à cárie dental durante a formação acadêmica. Esse dado é preocupante, uma vez que a cárie dental é a doença bucal com maior prevalência no mundo e pode ser evitada ${ }^{12,13}$.
Pouco mais da metade $(60,5 \%)$ dos docentes afirmaram abordar o tema cárie em sala de aula, dados semelhantes aos de Costa et al. ${ }^{14}$, os quais verificaram que $59 \%$ dos professores do ensino fundamental relataram transmitir informações acerca da saúde bucal aos alunos. Além disso, o presente estudo ainda evidenciou que professores de escolas públicas abordam menos o tema durante as 
aulas. Uma vez que a cárie apresenta distribuição desigual com polarização entre indivíduos com piores condições socioeconômicas ${ }^{15}$, o dado reforça a necessidade de inclusão dessa temática no sistema educacional brasileiro. O Ministério da Saúde ${ }^{16}$ reconhece que, além da escola ter uma função pedagógica que lhe é específica, ela tem uma função social e política voltada para a transformação da sociedade. Desta maneira, destaca-se a importância em se lecionar conteúdos relacionados à saúde no ambiente escolar. No entanto, sistematicamente não há a inclusão de temas como a cárie dental nas diretrizes curriculares.

Os docentes desse estudo apresentaram conhecimento básico sobre a cárie dentária, resultado semelhante ao encontrado no estudo de Aragão et $a l .{ }^{17}$, em creches municipais de João Pessoa/PB. Ainda assim, os docentes do presente estudo afirmaram que a maior parte do conhecimento adveio de informações dadas pelo cirurgião-dentista no consultório, conforme também encontrado em estudo com acadêmicos ${ }^{9}$ e professores $^{18}$. Nesse aspecto, vale ressaltar a importância do cirurgião-dentista na educação em saúde bucal durante sua prática clínica ${ }^{18}$. Outros locais relatados como fonte de informações foram em casa, no âmbito familiar e no ambiente escolar, semelhante aos dados obtidos em amostra de acadêmicos de Odontologia - 17,4\% dos participantes relataram que a maior parte do conhecimento sobre cárie veio da escola ${ }^{9}$. Esses dados demonstram a importância de professores bem capacitados sobre o tema para a realização da educação em saúde durante período de formação de um indivíduo, facilitando a mudança ou implementação de atitudes, hábitos e cuidados com a própria saúde bucal, já que esse desafio não precisa ser exclusivo do cirurgião-dentista ${ }^{9}$.

Embora a maior parte dos professores soubesse conceituar a cárie como uma doença, ainda assim houve dúvida quanto às consequências do desenvolvimento da mesma, já que 39,5\% apontaram ser uma lesão e 31,6\% responderam que resulta em perda dentária. O mesmo foi observado quando a maioria $(55,3 \%)$ afirmou a necessidade de sempre restaurar a cárie. A falta de informações sobre a necessidade ou não de tratamento restaurador demonstra a importância de se trabalhar com os professores que a cárie, em seu estágio inicial, se apresenta como uma lesão de mancha branca passível de remineralização, possibilitando sua remissão sem a necessidade de restauração ${ }^{19}$. Mesmo em estágios mais avançados, como a formação de uma cavidade, dependendo da sua extensão e possibilidade de limpeza por meio da escovação dentária, remoção de placa e uso de fluoretos, o processo de doença pode ser paralisado sem necessidade de intervenção restauradora ${ }^{20}$. Assim, verifica-se que um professor melhor informado pode contribuir de maneira mais efetiva no processo ensino-aprendizagem sobre saúde nas escolas $^{21}$.

O conhecimento a respeito do tema também pode ser observado quando se questiona sobre os métodos de prevenção da cárie. A grande maioria dos professores da amostra conhece e utiliza a escova e o fio dental, já 84,2\% relataram conhecer os cuidados com a alimentação e $73,7 \%$ o uso de bochechos com flúor, sendo que esses métodos são utilizados por apenas 50,0\% e 55,3\% dos respondentes, respectivamente. Tanto a alimentação quanto o uso do flúor desempenham papel fundamental no processo de desenvolvimento de lesões de cárie, já que uma dieta rica em sacarose favorece o estabelecimento e proliferação de microrganismos cariogênicos, tornando o biofilme mais espesso e aumentando consequentemente os períodos de desmineralização dental ${ }^{1}$. Na ausência de fluoreto, o esmalte se torna mais susceptível a desmineralização, possibilitando a instalação do processo carioso e o surgimento de lesões iniciais de mancha branca ${ }^{22}$.

A visita periódica ao dentista, conhecida por 94,7\% dos docentes, só é realizada regularmente por 
$76,3 \%$ deles, com periodicidade anual para $47,4 \%$ dos respondentes. O retorno periódico ao cirurgiãodentista é recomendado por possibilitar a preservação e manutenção da saúde bucal, para que as lesões iniciais de cáries possam ser diagnosticadas, permitindo o exercício de uma Odontologia minimamente invasiva e preventiva ${ }^{23}$. Vale ressaltar que no presente estudo todos os docentes possuem nível superior, 81,6\% deles com curso de pós-graduação, caracterizando alto nível de escolaridade. A renda dos participantes variou em sua maioria entre 5 e 6 salários mínimos, esta informação é importante, uma vez que há associação entre melhores práticas de saúde bucal e as condições financeiras dos docentes ${ }^{24}$.

Apesar do declínio da prevalência da cárie em crianças e adolescentes brasileiros nas últimas décadas, a doença ainda é um problema de saúde pública $^{2}$. Nos últimos anos, a Odontologia tem conseguido grandes avanços no que diz respeito aos conhecimentos técnico-científicos e à prevenção das doenças bucais, contudo estas ainda acometem grande parcela da população ${ }^{13}$. Embora os benefícios da mudança de hábitos de vida sejam amplamente conhecidos pelos profissionais, nem sempre são de conhecimento da população em geral $^{11}$. Sendo ainda o serviço odontológico limitado, sem cobertura total de Equipes de Saúde Bucal, e o número de profissionais insuficiente para acelerar o processo de reversão do modelo assistencialista para o de atenção à saúde, as ações intersetoriais com a educação podem ser importantes para a redução do impacto da cárie dentária na população brasileira.

Assim, deve-se ressaltar que uma ação integradora entre educação e Odontologia, introduzindo-se efetivamente o conteúdo saúde bucal no currículo do ensino infantil, fundamental e médio, viabilizaria formar crianças e adolescentes com perfil diferenciado, aptos a aplicar os conhecimentos adquiridos de forma mais profunda dentro do contexto de cada faixa etária e a realizar sua própria promoção de saúde bucal. Tornar todas as escolas promotoras de saúde bucal é uma meta a ser alcançada, visto que um professor bem informado, qualificado e estimulado poderá ser bem utilizado como agente multiplicador de saúde bucal.

Uma das limitações do estudo foi a perda amostral, principalmente pela dificuldade de permissão da realização do estudo nas escolas e a baixa devolutiva dos questionários preenchidos, o que pode excluir os indivíduos que apresentavam menor ou maior nível de informação ou conhecimento sobre o assunto. Da mesma forma, os resultados obtidos em uma cidade específica não podem ser extrapolados para a população. No entanto, o estudo trás dados inéditos e relevantes para discussão da inserção do tema cárie no sistema educacional brasileiro.

\section{CONCLUSÃO}

A maioria dos docentes pesquisados não recebeu informações a respeito do processo saúde-doença cárie durante curso de graduação, e sim a partir do dentista particular, de casa e da escola. Todos afirmaram que houve melhora da própria saúde bucal a partir da aplicação do conhecimento adquirido e a grande maioria dos docentes soube definir cárie como uma doença, contudo houve relativo desconhecimento de suas consequências e formas de tratamento.

\section{AGRADECIMENTO}

Agradecemos o financiamento da Fundação de Amparo à Pesquisa e Inovação do Espírito Santo (Fapes) através da participação do Programa de Bolsas de Iniciação Científica (PIBIC) da Universidade Federal do Espírito Santo (2016/2017).

\section{ABSTRACT \\ Knowledge about dental caries among high school teachers}

The objective of this study was to evaluate the 
knowledge about dental caries among high school teachers. This was a transversal descriptive study with a quantitative approach among high school teachers from public and private schools in the city of Vitória/ES, Brazil. The study used a validated self-administered questionnaire adapted for the target population with questions about the knowledge of caries. The descriptive data were presented in absolute and relative frequencies and the association test with Fisher's Exact test ( $\mathrm{p}<0.05)$. A total of 38 teachers participated in the study, $25(65.8 \%)$ of public schools. Most teachers did not receive information about caries $(60.5 \%)$ during their academic training and their knowledge was acquired during visits to the private dentist $(50.0 \%)$. Most of them knew how to define caries as a disease $(71.1 \%)$, however, there was uncertainty about whether the lesion $(39.5 \%)$ was the main consequence of its development and the obligatoriness of need for restoration $(55.3 \%)$ for its treatment. The caries theme is addressed in the classroom by $60.5 \%$ of teachers, mainly in the second year $(60.9 \%)$ and in private schools $(\mathrm{p}=$ 0.0285). Most part of the teachers would like that caries become part of the curricular structure of secondary education $(92.1 \%)$ as they believe in the relevance of this subject $(97.4 \%)$. As a conclusion, most teachers knew the definition of caries as a disease, however, there was relative ignorance of its consequences and ways of treatment.

Descriptors: Dental Caries. Health Education. Education, Primary and Secondary.

\section{REFERÊNCIAS}

1. Petry PC, Pretto SM. Educação e motivação em saúde bucal. In: Kriger L. (Org.) Promoção de saúde bucal. São Paulo: Artes Médicas, 2003. p.371-85.

2. Castilho ARF, Mialhe FL, Barbosa TS, Rontani RMP. Influência do ambiente familiar sobre a saúde bucal de crianças: uma revisão sistemática. J Pediatr. 2013; 89 (2):116-23.
3. Dalto V, Ferreira ML. Os professores como agentes promotores de saúde bucal. Semina. 1998; 19: 47-50.

4. Vasconselos RMML, Pordeus IA, Paiva SM. Escola: um espaço importante de informação em saúde bucal para a população infantil. Pós-Grad. Rev Fac Odontol. 2001; 4(3): 438.

5. Martins VR, Abrantes FN, Miasato JM. Professores como uma importante fonte de informação e promoção de saúde bucal. Pesq Bras Odontoped Clín Integr. 2008; 8(1): 27 30.

6. Ribeiro DG, Dovigo LN, Silva SRC. Avaliação de um método educativo em saúde bucal aplicado em escolares de ensino público. Arq Odontol. 2009; 45(3): 154-9.

7. Ferraz CA, Barros CS, Vieira LB. Utilização de instrumentos para avaliar ações educativas em saúde bucal: um relato de experiência universitária. Rev Baiana Saúde Pública. 2009; 33(2): 204-13.

8. Garcia PPNS, dos Santos PA, Castro CF, Oliveira ALBM, Dotta EAV. Conhecimento de cárie dental e doença periodontal de professores do ensino fundamental segundo o tipo de instituição (pública ou privada). Odonto. 2010;18(36):155-63.

9. Santos-Daroz CB, Santos GF, Silva-Junior MF, Gavi RS, Gomes MJ, Daroz LGD. Avaliação do conhecimento prévio e adquirido sobre cárie dentária em graduandos de Odontologia da Universidade Federal do Espírito Santo. Arq Odontol. 2016; 52(1): 23-31.

10. Campos L, Bottan ER, Farias J, Silveira EG. Conhecimento e atitudes sobre saúde e higiene bucal dos professores do ensino fundamental de Itapema-SC. Rev Odontol Unesp. 2008;37(4):389-94.

11. Ferreira JMS, Massoni ACLT, Forte FDS, Sampaio FC. Conhecimento de alunos 
concluintes de Pedagogia sobre saúde bucal. Interface Comunic Saúde Educ. 2005; 9(17): 381-8.

12. Kassebaum NJ, Bernabé E, Dahiya M, Bhandari B, Murray CJ, Marcenes W. Global burden of untreated caries: a systematic review and metaregression. J Dent Res. 2015;94(5):650-8.

13. Kassebaum NJ, Smith AGC, Bernabé E, Fleming TD, Reynolds AE, Vos T, Murray CJL, Marcenes W et al. Global, regional, and national prevalence, incidence, and disability-adjusted life years for oral conditions for 195 Countries, 1990-2015: A Systematic Analysis for the Global Burden of Diseases, injuries, and risk factors. J Dent Res. 2017;96(4):380-7.

14. Costa MM, Barbosa ADL, Fernandes JMFA, Fonseca FRA, Paredes SO. Conhecimento e práticas em saúde bucal nas escolas de ensino fundamental de um município de pequeno porte do sertão paraibano. Arq Odontol. 2014; 50(4):193202.

15. Narvai PC, Frazão P, Roncalli AG, Antunes JLF. Dental caries in Brazil: decline, polarization, inequality and social exclusion. Rev Panam Salud Publica. 2006;19(6):38593.

16. Brasil. Ministério da Educação e Cultura. Secretaria de Educação Fundamental. Parâmetros Curriculares Nacionais: terceiro e quarto ciclos: apresentação dos temas transversais. Brasília: Ministério da Educação e Cultura. p.436, 1998.

17. Aragão AKR, Sousa PGB, Ferreira JMS, Duarte RC, Menezes VA. Conhecimento de professores das creches municipais de João Pessoa sobre saúde bucal infantil. Pesq Bras Odontoped Clin Integr. 2010; 10(3):393-8.
18. Antunes LS, Antunes LAA, Soraggi MBS, Maia LC, Corvino MPF. Auto-avaliação, conhecimento e práticas de professores e agentes de educação frente a saúde bucal. Braz Oral Res. 2006; 20:170.

19. Maltz M, Tenuta LMA, Groisman S, Cury JA. Cariologia: Conceitos básicos, diagnóstico e tratamento não restaurador. São Paulo: Artes Médicas, 2016.

20. Maltz M, Jardim JJ, Alves LS. Health promotion and dental caries. Braz Oral Res. 2010; 24(Suppl. 1):18-25.

21. Franchin V, Basting RT, Mussi AA, Flório FM. A importância do professor como agente multiplicador de Saúde Bucal. Rev ABENO. 2006; 6(2):102-8.

22. Buzalaf MAR. Fluoretos e Saúde Bucal. São Paulo: Santos, 2008.

23. Tumeras I, Pascottos R, Saade JL, Bassani M. Odontologia minimamente invasiva. Rev Assoc Paul Cir Dent. 2014; 68(4):283-95.

24. Manjunath G, Kumar NN. Oral health knowledge, attitude and practices among school teachers in Kurnool - Andhra Pradesh. JOHCD. 2013; 7(1):17-24.

\section{Correspondência para:}

Cláudia Batitucci dos Santos Daroz e-mail:claudiabatitucci@yahoo.com.br Av. Marechal Campos 1468, Maruípe 29040-090 Vitória/ES 